\title{
AUTHORS' REPLY: INFLUENZA VACCINATION COVERAgE IN THE UNITED KINGDOM
}

\author{
P R Blank (patricia.blank@ifspm.uzh.ch) ${ }^{1}$, M Schwenkglenks ${ }^{2}$, T D Szucs ${ }^{1}$ \\ 1. Institute of Social- and Preventive Medicine, University of Zurich, Zurich, Switzerland \\ 2. European Center of Pharmaceutical Medicine, University of Basel, Basel, Switzerland
}

\section{Dear Editor,}

In response to our study investigating influenza vaccination coverage rates in the United Kingdom over six seasons with a telephone-based methodology [1], Pebody and colleagues note differences between our results and those reported by the Health Protection Agency (HPA) on behalf of Department of Health for registered patients in general practices in England. In their letter, Pebody and colleagues focus on our finding of a decline in coverage from $78.1 \%$ to $65.3 \%$ in the population aged 65 or over between 2005-6 and 2006-7.

At a closer look, our coverage results for this age group and those reported by the HPA are quite similar for $2003-4$ (70\% vs. $71 \%$, respectively) and $2005-6$ (78\% vs. $75 \%$, respectively) while our coverage results for 2004-5 and 2006-7 are indeed substantially lower. Given confidence interval widths, as shown in Figure 2 of our report, chance alone does not appear to be a likely explanation for this discrepancy.

With respect to selection effects, it should be noted that our survey methodology was designed to capture a representative sample of the population, even in the presence of low response rates. We are aware, however, that the increasing use of answering machines, voicemail systems, caller IDs and mobile phones creates an emerging challenge for telephone surveys [2,3]. Given that, we clearly cannot exclude selection bias as a partial explanation. However, as our methodology remained the same during the entire time period covered, it is unclear why selection bias should have occurred in two of the influenza seasons covered, but not in the other two.

The interviews were generally conducted between December and February as most individuals get (typically) vaccinated between September and November. The time lag between the vaccination and the fieldwork period was kept small in order to minimise incorrect recall of vaccination status. However, if the time distribution of vaccination episodes is atypical, as may have been the case in the 2006-7 influenza season due to delayed availability of the vaccine, this approach to survey timing may potentially lead to underreporting.

It should also be noted that our household surveys do not study the same population as the HPA. While our methodology covers the entire population that can be reached by a (landline) telephone connection, the HPA approach is restricted to those persons who are registered with a general practitioner and hence will, on average, differ in health status and perhaps other respects. Our knowledge of the British health system is not detailed enough to make a judgement on the potential implications of this difference.

In order to avoid double counting, we primarily studied only those persons aged 65 or over who did not have a chronic illness and were not working as health care professionals, i.e. we did not include all persons aged 65 or over, as was the case in the HPA analyses. Lower coverage rates would be expected for the resulting lower risk group, while in those aged 65 or over and with a chronic disease, whom we analysed separatly, distinctly higher coverage rates were indeed seen.

In conclusion, it is not obvious, in our opinion, what gave rise to the differences noted by Pebody and colleagues. There are several possible explanations which are not mutually exclusive. The different approaches to studying influenza coverage rates may be complementary rather than contradictory.

\section{References}

1. Blank PR, Freiburghaus AU, Schwenkglenks M, Szucs TD. Trends in influenza vaccination coverage rates in the United Kingdom over six seasons from 2001-2 to 2006-7. Euro Surveill. 2008;13(43):pii=19014. Available from: http:// www.eurosurveillance.org/ViewArticle.aspx?ArticleId $=19014$

2. Feveile $\mathrm{H}$, Olsen $\mathrm{O}$, Hogh $\mathrm{A}$. A randomized trial of mailed questionnaires versus telephone interviews: response patterns in a survey. BMC Med Res Methodol. 2007;7:27.

3. Kempf AM, Remington PL. New challenges for telephone survey research in the twenty-first century. Annu Rev Public Health. 2007;28:113-26.

This article was published on 18 December 2008.

Citation style for this article: Blank PR, Schwenkglenks M, Szucs TD. Authors reply: Influenza vaccination coverage in the United Kingdom. Euro Surveill. 2008;13(51):pii=19075. Available online: http://www.eurosurveillance.org/ViewArticle. aspx?ArticleId $=19075$ 